Gut, 1985, 26, 961-967

\title{
Liver and biliary
}

\section{Computer-aided selection of diagnostic tests in jaundiced patients}

\author{
M-F SAINT-MARC GIRARDIN, M LE MINOR, A ALPEROVITCH, \\ F ROUDOT-THORAVAL, J-M METREAU, AND D DHUMEAUX \\ Service d'Hépatologie et de Gastroentérologie, Unité de Recherches INSERM 99, Hôpital Henri Mondor, \\ Créteil, France, and Unité de Recherches INSERM 169, Villejuif, France
}

SUMmARY A model has been developed for ordering diagnostic tests in jaundiced patients. The system proceeds in two steps: (i) diagnostic hypotheses are calculated for each patient from the results of physical examination and routine biological investigations; (ii) given these hypotheses, the most efficient test (out of 22) for reaching the final diagnosis is selected using four criteria: diagnostic value, risk, financial cost, and time in obtaining the result. This model was tested in 62 patients. In 43 of them (69\%), the selected test was sufficient for reaching a diagnostic accuracy of $100 \%$. In this group of patients, a mean of 3.7 (range 1-6) tests per patient was ordered by physicians. In the 19 remaining patients, the selected test was not sufficient for the final diagnosis, thus requiring a multiple choice process. It is suggested that such a system could help physicians to improve the care of patients by more efficient ordering of diagnostic tests.

During the last decade, impressive advances in medical technology have provided many new diagnostic tests. Because of the large number of tests now available, the main difficulty for physicians today is not to reach the final diagnosis but to define the most efficient approach for the final diagnosis. In recent years, the cost related to testing has become a major factor in the growth of health care expenses, and many publications have focused on the selection, ${ }^{1-4}$ and on the cost effectiveness ${ }^{56}$ of diagnostic tests.

For correct ordering of tests, physicians should theoretically have in mind precise and up to date information about all relevant tests, in particular their sensitivity, specificity, potential risk of morbidity and mortality, and financial cost. Obviously, this ideal situation is rarely attained, and it has been postulated that difficulties in managing patients are partly because of man's inability to handle many different parameters simultaneously. ${ }^{78}$ Because they do not have such a limitation, computers were thought to provide valuable help in this field. The model that we have developed concerns patients hospitalised for jaundice of unknown aetiology. Its aim is to select in these patients the 'best' diagnostic test - that is, the test which is expected to lead

Address for correspondence: M-F Saint-Marc Girardin, INSERM U-99, Hôpital Henri Mondor, 94010 Créteil, France.

Received for publication 30 October 1984 directly to the final diagnosis, within a cost effective framework. Results obtained for 62 patients suggest that this model is capable of correctly selecting the appropriate diagnostic tests in jaundiced patients and could help clinicians to reduce the number of tests.

\section{Methods}

DESCRIPTION OF THE MODEL

The model derives in part from a computer aided system that we previously developed for the diagnosis of diseases of the liver and biliary tract. ${ }^{9}$ In the present study, its utilisation was limited to patients with conjugated hyperbilirubinaemia. The differential diagnosis was made from 36 hepatobiliary diseases which together represent more than $95 \%$ of all the diseases possibly associated with conjugated hyperbilirubinaemia. The $a$ priori and the conditional probabilities, which act on the model through the use of Bayes' theorem, were estimated as detailed in previous publications. ${ }^{-11}$ These data were provided from a sample of more than 1000 patients with liver disease. The model includes two successive steps: in the first step, the diagnostic probabilities are calculated for each patient from the following parameters: (i) 30 clinical signs (19 from patient's history and 11 from clinical examination); (ii) 21 biological informations resulting from routine haematologic 
and liver tests, which are always available within the first six hours of admission. More complex diagnostic tests were purposely not included at this step. In the second step, the selection of the most efficient test for obtaining the final diagnosis is computed from the 22 diagnostic tests listed in Table 1 . These tests are those most commonly used for diagnosing patients with conjugated hyperbilirubinaemia. Four criteria characterising each diagnostic test were used for programing the selection: diagnostic value, potential risk, financial cost and time in obtaining the result. Whereas the values of financial cost and delay in obtaining the result are identical whatever the disease, diagnostic value and risk are disease dependent. Thus, the values of the last two parameters were calculated for each patient according to the diagnostic probabilities computed at the first step of the model. In order to estimate the diagnostic value of the 22 tests, the conditional likelihood of each of them has been evaluated and quantified from 0 to 3 depending on its diagnostic value for each of the 36 diseases considered. This information was derived from literature and from the opinion of expert hepatologists. In the model, the diagnostic value of each test was calculated from a classical measure of uncertainty from the formula: $\mathrm{H}=-\Sigma \mathrm{P}_{\mathrm{j}}$ $\log P_{j}$ where $H$ expresses the uncertainty after the test and $P_{j}$ the probability of the disease $j$; thus, the lower the diagnostic uncertainty after the test, the better the value of the test. The risk of testing was

Table 1 List of the 22 diagnostic tests taken into account in the computer system

\begin{aligned} & \hline No Test \\ & \hline 1 Serologic tests for brucellosis \\ & 2 Serologic tests for infectious mononucleosis \\ & 3 Serum amylase \\ & 4 Serologic tests for amoebiasis \\ & 5 Plain radiograph of the abdomen \\ & 6 Serum non-specific tissue antibodies \\ & 7 Percutaneous liver biopsy* \\ & 8 Ultrasonography of the abdomen \\ & 9 Serum alpha fetoprotein \\ & 10 Serum ferritin \\ & 11 Serologic test for hydatid disease \\ & 12 Serologic test for liver fluke infection \\ & 13 Peritoneoscopy* \\ & 14 Lymph node surgical biopsy* \\ & 15 Mini-laparotomy* \\ & 16 Serologic tests for A and B virus \\ & 17 Hepatic scintiscan \\ & 18 Transvenous liver biopsy* \\ & 19 Computed axial tomography of the abdomen* \\ & 20 Endoscopic retrograde cholangiography* \\ & 21 Percutaneous cholangiography* \\ & 22 Hepatic arteriography* \\ & \hline\end{aligned}

* Tests with a potential risk. also estimated from published data and by expert hepatologists and was also quantified from 0 to 3 . Among these 22 diagnostic tests, no risk existed for 13 and a varying degree of risk existed for 9 (Table 1). For tests with a potential risk, the degree of the risk depended either on the diagnostic hypotheses or on the coexistence of an abnormal sign (abnormality of clotting factors for example). The financial cost of the 22 diagnostic tests was established by examining data from the National Health Insurance scheme for patients admitted to Henri Mondor Hospital. The time in obtaining the result was also established for patients hospitalised in our hospital. For 15 out of 22 tests, this delay was less than seven days.

The model used for the selection of the diagnostic tests ranks each of them from best to worst according to a score computed by aggregation of the four criteria listed above. For this purpose, each of the four criteria has been weighted. A weight (w) of 0.4 was arbitrarily assigned to both diagnostic value and risk. For financial cost and time in obtaining the result, the value of $w$ was $0 \cdot 1$. For each test (i), the global score $\left(\mathrm{C}_{\mathrm{i}}\right)$ was computed from the formula:

$$
C_{i}=\sum_{j=1}^{36} P_{j} \cdot \sum_{k=1}^{4} w k C_{i j k}
$$

where $P_{j}$ expresses the probability of the disease $j$, wk the weight of the criteria $\mathrm{k}$, and $\mathrm{C}_{\mathrm{ijk}}$ the value of criteria $\mathrm{k}$ for the test $\mathrm{i}$ if the disease is $\mathrm{j}$. Thus, the first test selected was that which had the lowest calculated global score.

EVALUATING THE MODEL

The selection of the diagnostic tests was retrospectively studied in 62 patients hospitalised consecutively in our liver unit for evaluation of conjugated hyperbilirubinaemia. There were 35 men and 27 women, ranging in age from 17 to 89 years, the average being 50 years. In each case the final diagnosis was firmly established (liver histology, laparotomy, necropsy, or prolonged clinical followup) (Table 2).

The results were analysed: (i) in comparing the test proposed by the model to those carried out by the clinicians who had previously managed the patients; (ii) in evaluating the diagnostic efficacy of

Table 2 Final diagnosis in the 62 studied patients

\begin{tabular}{lrll}
\hline Acute viral hepatitis & 25 & Primary biliary cirrhosis & 1 \\
Alcoholic hepatitis & 3 & Hepatocellular carcinoma & 7 \\
Chronic active hepatitis & 2 & Secondary liver cancer & 5 \\
Alcoholic cirrhosis & 6 & Neoplastic biliary & \\
Cryptogenic cirrhosis & 2 & obstruction & 5 \\
Post-hepatitic cirrhosis & 1 & Choledocolithiasis & 5 \\
\hline
\end{tabular}


the computed test according to the final diagnosis.

\section{Results}

\section{DIAGNOSTIC PROBABILITIES COMPUTED FROM BASIC INDICANTS}

Examination of the diagnostic hypotheses computed at the first step of the model with only basic information showed that the model correctly discriminated patients with intrahepatic from those with extrahepatic jaundice: in fact, a correct separation according to the site of jaundice was achieved in 59 out of 62 patients $(95 \%)$. Not surprisingly, however, the performances were lower for diagnosing the precise cause of jaundice; this was achieved in only 34 patients $(54 \%)$. In addition, among these 34 patients, a confident diagnosis (probability $\geqslant 0.95$ ) was made by the computer in only 20 cases. These results are due to the fact that in most cases, the basic information available at the first step was not sufficient to reach a final diagnosis, supporting the utility of computer aided selection of diagnostic tests.

\section{SELECTION OF DIAGNOSTIC TESTS}

(1) Characteristics of the selected diagnostic test

The list of diagnostic tests proposed by the model in the 62 patients is shown in Table 3. For each patient, the test selected by the computer from the global score was compared with the test that the computer would have selected by considering only the di-

Table 3 Tests selected by the program according to the global score or the diagnostic value alone

\begin{tabular}{|c|c|c|}
\hline & $\begin{array}{l}\text { Test selected } \\
\text { from global } \\
\text { score }\end{array}$ & $\begin{array}{l}\text { Test selected } \\
\text { from diagnostic } \\
\text { value alone }\end{array}$ \\
\hline \multicolumn{3}{|l|}{ Serologic tests for $\mathbf{A}$ and } \\
\hline B virus & 19 & 15 \\
\hline \multicolumn{3}{|l|}{ Ultrasonography of the } \\
\hline abdomen & 13 & 1 \\
\hline Peritoneoscopy & 6 & 5 \\
\hline Serum alpha, fetoprotein & 4 & 2 \\
\hline Percutaneous liver biopsy & 4 & 6 \\
\hline $\begin{array}{l}\text { Computed axial tomography of } \\
\text { the abdomen }\end{array}$ & 4 & 7 \\
\hline Transvenous liver biopsy & 3 & 10 \\
\hline Mini-laparotomy & 2 & 11 \\
\hline \multicolumn{3}{|l|}{ Serum non-specific tissue } \\
\hline antibodies & 2 & 1 \\
\hline Serum amylase & 2 & 0 \\
\hline \multicolumn{3}{|l|}{ Serologic test for } \\
\hline mononucleosis & 2 & 1 \\
\hline Serum ferritin & 1 & 0 \\
\hline Hepatic arteriography & 0 & 3 \\
\hline Total number of patients & 62 & 62 \\
\hline
\end{tabular}

agnostic value. These tests were identical in 43 patients and differed in the 19 others. The clearest difference was observed for three tests: ultrasonography, transvenous liver biopsy and minilaparotomy (Table 3): the first was more frequently selected when the global score was taken into account, and the latter two when considering the diagnostic value alone. These results suggest that in this study the weight assigned to the diagnostic value does not play an excessive role in the evaluation of the global score and can be counterbalanced by other criteria.

(2) Comparison of the diagnostic test selected by the computer with those ordered by the physicians

The test selected by the computer was among those ordered by the clinicians who had previously managed the patients in 41 out of the 62 patients $(66 \%)$. The best concordance was observed in patients with viral hepatitis, cirrhosis and biliary obstruction: the test selected by the model was ordered by clinicians in $23 / 30,8 / 10$ and $7 / 10$ of these patients, respectively. In contrast, a poor concordance between the physicians and the model was observed in patients with liver carcinoma, since a discordance was observed in $9 / 12$ patients.

\section{(3) Efficacy of the selected diagnostic test in reaching the final diagnosis}

The diagnostic efficacy of the test was assessed by studying the number of cases in which the test selected by the model would have been sufficient for reaching the final diagnosis. In the 41 patients in whom the diagnostic test selected by the computer was carried out by the physicians, its diagnostic efficacy could be easily evaluated. Efficacy was proven in 29 out of these 41 patients. In the 21 other patients, the diagnostic test proposed was not among those done by the physicians. In these patients, efficacy of the diagnostic test was evaluated indirectly by considering the likelihood of its result based on the final diagnosis. Using this method, we found that the selected test, if undertaken, would have led to the final diagnosis in 14 out of the 21 patients.

In conclusion, two groups of patients could be distinguished from the results of the computed selection (Table 4): Group 1 included the 43 patients in whom the diagnostic test either did or would have led to the correct final diagnosis. This group was mainly composed of patients with typical viral hepatitis, biliary obstruction and liver carcinoma. Group 2 included the 19 patients in whom the diagnostic test, alone, would not have led to the final diagnosis. This group was mainly composed of patients with non-typical hepatitis and cirrhosis, and 
Table 4 Diagnostic efficacy of the computed selection in the 62 patients

\begin{tabular}{lll}
\hline & $\begin{array}{l}\text { Group 1 } \\
\text { (43 pts) }\end{array}$ & $\begin{array}{l}\text { Group 2 } \\
\text { (19 pts) }\end{array}$ \\
\hline Hepatitis (30 pts) & 22 & 8 \\
Cirrhosis (10 pts) & 3 & 7 \\
Liver carcinoma (12 pts) & 9 & 3 \\
Biliary obstruction & & \\
$\quad$ Choledocolithiasis (5 pts) & 4 & 1 \\
$\quad$ Neoplastic obstruction (5 pts) & 5 & 0 \\
\hline
\end{tabular}

Group 1 included patients in whom the selected test either did or was expected to have led to the final diagnosis (see text for details). Group 2 included patients in whom the selected test would not have led to the final diagnosis (see text for details).

could be further separated into three categories: (i) Four patients for whom the computed selection was correct by reference to the final diagnosis but in fact failed to reach it due to a false negative result. (ii) Ten patients for whom the computed selection was again correct, but in whom the final diagnosis required necessarily more than one test. (iii) Five patients for whom the final diagnosis actually required another diagnostic test than that selected by the program. In three out of these five patients, the final diagnosis was not among the main diagnostic hypotheses computed at the first step. As the diagnostic tests were selected from these hypotheses, this explains the inadequate selection of the model in these cases.

Although a comparison between computer and physicians was not the aim of this study, we examined the number of tests actually undertaken by physicians for each patient of the two groups (Table 5). In Group 1 (in which the computed test alone would have been sufficient) the number of tests ranged from one to six. This number ranged from two to seven in Group 2.

Table 5 Number of tests performed by clinicians in the 62 patients

\begin{tabular}{|c|c|c|c|c|}
\hline & $\begin{array}{l}\text { Group } \\
\text { mean }\end{array}$ & $\begin{array}{r}(43 \mathrm{pts}) \\
\text { (range) }\end{array}$ & $\begin{array}{l}\text { Group } \\
\text { mean }\end{array}$ & $\begin{array}{r}2(19 \text { pts }) \\
\text { (range) }\end{array}$ \\
\hline Hepatitis & $2 \cdot 1$ & $(1-4)$ & $3 \cdot 1$ & $(2-4)$ \\
\hline Cirrhosis & $4 \cdot 3$ & $(4-5)$ & $4 \cdot 1$ & $(3-6)$ \\
\hline Liver carcinoma & $3 \cdot 4$ & $(2-5)$ & $5 \cdot 3$ & $(4-7)$ \\
\hline \multicolumn{5}{|l|}{ Biliary obstruction } \\
\hline Choledocolithiasis & $3 \cdot 7$ & $(4-5)$ & $5^{*}$ & \\
\hline Neoplastic obstruction & $5 \cdot 0$ & $(4-6)$ & & \\
\hline
\end{tabular}

Group 1 included patients in whom the selected test either did or was expected to have led to the final diagnosis (see text for details). Group 2 included patients in whom the selected test would not have led to the final diagnosis (see text for details).

* Only one patient had choledocolithiasis in this group.
Discussion

The large number of diagnostic procedures now available in medicine probably contributes to improve the performances of physicians, but certainly increases the financial cost of testing. Computers, with their ability to store large amounts of data and to carry out complex operations, have been shown to be of interest in various areas of medical diagnosis. ${ }^{9}{ }^{12-15}$ Thus, it could be assumed that they might also optimise the process of ordering of diagnostic tests. To our knowledge, the model reported here represents one of the first examples of computer aided selection of diagnostic tests. We deliberately considered the problem of jaundice because of our previous experience of a computer aided system in hepatology, ${ }^{10}$ and also because of the practical importance of this problem: cholestatic jaundice is a frequent cause of admission and the optimum management of such patients, partly because of the multiplicity of diagnostic tests, remains problematic.

The methodology that we have adopted in this program closely mimics the diagnostic approach of physicians and proceeds in two steps. In the first step, using the Bayesian probability theory, the program proposes diagnostic hypotheses (expressed as probabilities) which are computed from clinical information and from the results of routine tests. In the second step, the model selects the diagnostic test which is expected to lead directly to the final diagnosis with the lowest global score. To formulate its choice, considering the initially computed diagnostic hypotheses, the model takes into account the expected diagnostic value and risk of the available tests. It also considers two important but often overlooked criteria: the financial cost of the test and the delay in obtaining its result. The latter parameter is of great interest because of its obvious interference with the speed of the diagnosis and consequently with the cost of hospital care. With the introduction of these financial criteria, the selection provided by the computer is expected to be costeffective, and this is in accordance with the recommendations of all policies dealing with health care. ${ }^{16-18}$ Among the criteria which we used, the patient's discomfort due to the tests as well as tests associating therapeutic usefulness with diagnostic value - for example, sphincterotomy combined with endoscopic retrograde cholangiography, were not taken into account. Although the importance of these factors is recognised there is too little information available at the present time in the literature to allow their quantification.

Our data suggest that the model which we have developed is able to provide an appropriate selec- 
tion of diagnostic tests and thus, could help physicians in their diagnostic process. First of all, the test selected for each patient appeared to be always coherent with the diagnostic hypotheses computed in the first step. In addition, when possible contraindications were checked, none of the selected tests was contra-indicated by these hypotheses. As concerns the nature of the tests chosen by the model, it is now well recognised that the first diagnostic test to be carried out in patients with cholestatic jaundice must be non-invasive $e^{19-22}$ and this is exactly the procedure followed by the model. This explains why percutaneous cholangiography or retrograde cholangiography, two procedures with a very high diagnostic value, were not chosen by the model as the first test. In contrast, as expected, ultrasonography of the abdomen was the most frequently selected test.

The selected test did (or was expected to) lead directly to the final diagnosis in 43 out of the 62 patients $(69 \%)$. This was observed mainly in biliary obstruction, liver carcinoma and viral hepatitis: indeed, in the first two conditions, the most frequently selected test (ultrasonography, computed axial tomography, peritoneoscopy) have a high diagnostic accuracy. As viral hepatitis is concerned, among the 25 cases included in this study, 15 were clinically typical and required only serological markers to be confirmed. In the 19 other patients $(41 \%)$, the test selected by the program failed to give directly the final diagnosis. This cannot necessarily be interpreted as a failure, because another diagnostic test should have been selected to reach the final diagnosis in only five of these patients. In 10 patients, suffering from cirrhosis or non-typical hepatitis, at least two tests were necessary to obtain the final diagnosis: for example, the diagnosis of patients with post-hepatitic cirrhosis requires not only liver biopsy, but also determination of hepatitis B virus markers. Finally, it is also well recognised that the information provided by diagnostic tests, even if properly ordered, is not perfect. A 'perfect' test is characterised by both sensitivity and specificity of $100 \%$; this obviously never occurs. Such a problem is illustrated by the four remaining patients of this group for whom the selected test was actually appropriate, but failed to give the final diagnosis because of false negative results.

The comparison between the tests selected by the computer and those carried out by the physicians in the same patients, shows that in 21 out of the 62 patients $(34 \%)$, the test selected by the model had not been ordered by the physicians. This was observed especially in the cases of liver carcinoma. At least two factors explain the lack of complete concordance: (i) several diagnostic tests, especially when liver carcinoma is suspected, have approximately the same diagnostic value and risk, but differ in their financial cost and delay in obtaining the result. The difference that we observed between computer and clinicians in such cases was due to the fact that the selection of the computer was more often cost-dependent than the one of physicians; (ii) in contrast with the clinician's approach, the model does not take into consideration possible changes in the patient's condition during hospital care. In such cases, diagnostic hypotheses may change and consequently, the diagnostic tests proposed may be different.

The greater number of tests ordered by physicians as compared with the computer can be explained in various ways: (i) It is clear that, when faced with a complex diagnostic problem, physicians, unlike the computer, may not imagine all the possible hypotheses and, consequently, are not in optimum conditions to select the best discriminating tests; (ii) new techniques, because attractive, are often ordered before their benefits or risks have been assessed properly. This results in old and new diagnostic tests being carried out on the same patient, without necessarily improving the diagnostic accuracy; (iii) hoping to reduce the time of diagnosis, physicians often prefer simultaneous ordering of a complete set of diagnostic tests to sequential ordering. At the present time, we do not know the best way to order diagnostic tests - that is, sequential (the computer's way) or global (the usual physicians' way) and studies comparing these two methods should be undertaken. Such a comparison between computer and physicians has, however, to be interpreted cautiously. In the present study, the selections made by the computer and the clinicians were compared retrospectively. In fact, when physicians managed their patients, they were not told that their plan of investigation would be subsequently compared with a computer model. If they had been advised of this, their diagnostic approach would perhaps have been different. It has in fact been shown that, when physicians are aware of the parallel use of a computer model, they are stimulated to improve their own clinical performance. ${ }^{23}$

Two criticisms of the present study must also be answered. Firstly, the retrospective study of patients prevented us from evaluating precisely the actual efficiency of the tests selected by our model. Ideally, this efficiency should be tested in a prospective way on patients randomly assigned to be managed either by the model or by physicians. Such a methodology, however, could not be ethically envisaged before having tested the safety of our system. Secondly, the accurate selection of diagnostic procedures clearly depends on the diagnostic hypotheses computed in 
the first step of the program. If these diagnostic hypotheses are not correct, the tests selected may be inadequate. In fact, it has been shown previously that the performance of the model at this step was equivalent to that of hepatologists (and significantly better than that of internists). ${ }^{9}$ Moreover, in the present study, the final diagnosis was included in the first four diagnostic hypotheses proposed by the computer, in $88 \%$ of all cases.

Although a number of computer aided diagnostic systems have been developed, only a few are actually used routinely. Several characteristics of our model lead us to suggest that it could be easily accepted and used by clinicians. As stated previously: (i) the model mimics their overall diagnostic approach; (ii) in contrast with most computer systems proposed for liver diseases, ${ }^{24-26}$ the number of possible diagnoses envisaged is large but the number of parameters used for the computation of the diagnostic hypotheses is low; (iii) finally, this model is one of the first which has been specifically developed for improving the selection of diagnostic tests: with potential exception for computed axial tomography of the abdomen, each of the diagnostic tests is now available in all medical institutions. Computerised selection of diagnostic tests was shown to be appropriate in the management of the patients considered in this study. Further studies are required, namely in other medical fields, before concluding that this quantitative technique represents the ideal way for improving patient's care by a more efficient ordering of diagnostic tests.

The authors are greatly indebted to Drs $\mathbf{R} P$ Knill-Jones, A M Preaux, M Goodhardt, and J L Goodhardt for their constructive contribution, and to Mrs C Petit and V Poli for their help in preparing the manuscript. This work has been supported by grants from the Direction du Plan de L'Assistance Publique à Paris, the Université Paris-Val de Marne, and the Institut National de la Santé et de la Recherche Médicale (CRL No 77-5-220-8).

\section{References}

1 Donaldson RM. Assessing the usefulness of diagnostic procedures. Gastroenterology 1977; 72: 762-3.

2 Griner PF, Mayewski RJ, Mushlin AI et al. Selection and interpretation of diagnostic tests and procedures. Principles and applications. Ann Intern Med 1981; 94: 553-600.

3 Griner PF, Glaser RJ. Misuse of laboratory tests and diagnostic procedures. $N$ Engl J Med 1982; 307: 1336-9.
4 Kassirer JP, Pauker SG. Should diagnostic testing be regulated? $N$ Engl J Med 1978; 299: 947-9.

5 Hull R, Hirsh J, Sackett DL et al. Cost effectiveness of clinical diagnosis, venography, and noninvasive testing in patients with symptomatic deep-vein thrombosis. $N$ Engl J Med 1981; 304: 1561-7.

$6 \mathrm{McNeil}$ BJ, Varady PD, Burrows BA et al. Measures of clinical efficacy. Cost-effectiveness calculations in the diagnosis and treatment of hypertensive renovascular disease. N Engl J Med 1975; 293: 216-21.

7 McDonald CJ. Protocol-based computer reminders, the quality of care and the non-perfectability of man. $N$ Engl J Med 1976; 295: 1351-55.

8 Myers JD. Preventing iatrogenic complications. $N$ Engl J Med 1981; 304: 664-5.

9 Begon F, Lockhart AM, Métreau JM et al. A computer-aided system for the diagnosis of hepato-biliary diseases. A comparison with the performance of physicians. Med Inform 1979; 4: 35-42.

10 Begon F, Dhumeaux D. The application of computer techniques to the laboratory diagnosis. Diseases of the liver and biliary system. Minn Med 1971; 54: 101-5.

11 Le Minor M, Alperovitch A, Lockhart AM et al. Strategy for the diagnosis of hepato-biliary disease: the computer-aided decision. Medcomp 77 Berlin. Uxbridge, England: Online Conferences Limited, 1977: 679-86.

12 De Dombal FT, Clamp SE, Leaper DJ et al. Computeraided diagnosis of lower gastrointestinal tract disorders. Gastroenterology 1975; 68: 252-60.

13 Goldman L, Weinberg M, Weisberg $\mathrm{M}$ et al. A computer-derived protocol to aid in the diagnosis of emergency room patients with acute chest pain. $N$ Engl J Med 1982; 307: 588-96.

14 Miller RA, Pople HE, Myers JD. Internist-I, an experimental computer-based diagnostic consultant for general internal medicine. $N$ Engl J Med 1982; 307: 468-76.

15 Fragu P, Alperovitch A, Patois E. Screening and diagnosis of hyperthyroidism: attempt at test reduction. $J$ Epidemiol Community Health 1979; 33: 215-8.

16 Hiatt HH. Protecting the medical commons: who is responsible? $N$ Engl J Med 1975; 293: 235-41.

17 Schwartz WB, Joskow PL. Medical efficacy versus economic efficiency: a conflict in values. $N$ Engl J Med 1978; 299: 1462-4.

18 Weinstein MC, Stason WB. Foundations of costeffectiveness analysis for health and medical practices. N Engl J Med 1977; 296: 716-21.

19 Dooley JS, Olney J, Dick R, Sherlock S. Non-surgical treatment of biliary obstruction. Lancet 1979; 2: 1040-4.

20 O'Connor KW, Snodgrass PJ, Swonder JE et al. A blinded prospective study comparing four current noninvasive approaches in the differential diagnosis of medical versus surgical jaundice. Gastroenterology 1983; 84: 1498-504.

21 Richter JM, Silverstein MD, Schapiro R. Suspected destructive jaundice: a decision analysis of diagnostic strategies. Ann Intern Med 1983; 99: 46-51.

22 Scharschmidt BF, Goldberg HI, Schmid R. Current concepts in diagnosis approach to the patient with 
cholestatic jaundice. $N$ Engl J Med 1983; 308: 1515-9.

23 De Dombal FT. Computer-aided diagnosis of abdominal pain. In: Biomedical computing. Baltimore: University Park Press, 1977: 351-8.

24 Burbank F. A computer diagnostic system for the diagnosis of prolonged undifferentiating liver disease. Am J Med 1969; 46: 401-15.
25 Knill-Jones RP, Stern RB, Girmes DH et al. Use of sequential Bayesian model in diagnosis of jaundice by computer. Br Med J 1973; 1: 530-3.

26 Stern RB, Knill-Jones RP, Williams R. Use of computer program for diagnosing jaundice in district hospitals and specialized liver unit. $B r \operatorname{Med} J$ 1975; 2: 659-62. 